\title{
Cyclooxygenase, Cancer Stem Cells and DNA Methylation Play Important Roles in Colorectal Carcinogenesis
}

\author{
Masahiko Tsujii \\ Department of Gastroenterology and Hepatology, Osaka University Graduate School of Medicine, Suita, Japan
}

\section{Key Words}

COX-2 $\cdot$ Colorectal cancer $\cdot$ Cancer stem cells .

Hypermethylation $\cdot$ Hypomethylation $\cdot$ Stemness

\begin{abstract}
Many effective anticancer therapies against colorectal cancer have been developed, but chemoresistance and recurrence are still inevitable problems and their counter measures are urgently needed. Recently, cancer stem cells have been indicated to play a pivotal role in chemoresistance and recurrence and have gained attention as a novel target. On the other hand, both aberrant hyper- and hypomethylation have been shown to be involved in carcinogenesis and the simultaneous amendment is indispensable. Cyclooxygenase-2 (COX-2) has already been reported to play an important role in carcinogenesis. Our latest study indicates that COX-2 inhibitors remedy aberrant methylation and beat cancer stemness, suggesting that COX-2 inhibitors hold great promise for cancer prevention and therapy.
\end{abstract}

Copyright $\odot 2013$ S. Karger AG, Basel
(C) 2013 S. Karger AG, Basel

0012-2823/13/0871-0012\$38.00/0

Fax +4161306 1234

E-Mail karger@karger.ch

www.karger.com
Accessible online at: www.karger.com/dig
Colorectal cancer (CRC) is the third most common cancer in the Western world. The 5-year survival rate is, at best, $10-20 \%$ for patients with distant metastatic disease. Currently, molecular target therapy, including cetuximab and bevacizumab, is used for the treatment of CRC in addition to FOLFOX or FOLFIRI chemotherapy. Cetuximab is an anti-epidermal growth factor receptor (anti-EGFR) monoclonal antibody, which binds to EGFR and turns off the uncontrolled growth in cancers with EGFR expression, showing a great antitumor effect. The overall response rate of a combination of cetuximab and FOLFIRI in the treatment of patients with unresectable CRC liver metastases is about $40 \%$, and the rate of conversion to resectable liver metastases is about $30 \%$, showing a survival advantage. However, KRAS mutations are found in approximately $40 \%$ of CRC cases, and such oncogenic activation of intracellular signaling pathways downstream of EGFR is an important mechanism of resistance to anti-EGFR antibodies. Therefore, the development of effective perioperative therapy is essential [1].

We have reported that cyclooxygenase-2 (COX-2) plays an important role in carcinogenesis [2]. COX-2 is overexpressed in most CRC tissues and produces prostaglandin E2 (PGE2). PGE2 enhances resistance to apopto- 
sis and the potential for invasion, angiogenesis, cell-proliferation, and lymph node and hematogenous metastasis, which favors carcinogenesis. COX-2 inhibitors are certified as useful chemopreventive agents and possible useful adjuvant remedies. PGE synthase, especially microsomal PGE synthase, and 15-hydroxyprostaglandin dehydrogenase (15-PGDH), a key enzyme catabolysing PGs, are attractive as novel molecular target drugs.

Many anticancer drugs for CRC have evolved, but the therapies are inherently limited by the inevitable recurrence of resistant tumor cells after initial responses. A putative explanation of ineffective therapy is the presence of cancer stem cells. Recently, this theory has been advocated even in solid tumor-like gastrointestinal tract cancer. Cancer stem cells are marked by characteristics such as self-renewal, pluripotency, tumor-initiating capacity, ATP-binding cassette transport, active DNA repair and resistance to chemotherapy and radiation therapy, which results in tumor recurrence [3].

On the other hand, CRC is influenced by epigenetic modification. A hallmark of cancer is the paradoxical copresence in the same tumors of local and global DNA hypomethylation together with the regional hypermethylation of certain genes. CpG hypermethylation suppresses tumor-suppressive genes and hypomethylation is involved in expression of genes related to carcinogenesis. Due to the oncogenic role of these different DNA methylation alterations, two therapeutic strategies are required: DNA-hypomethylating or DNA-demethylating agents (such as 5-aza-2-deoxycytidine) to abrogate the accumulation of hypermethylated genes and DNA-methylating agents (such as folate) to inhibit global or local DNA hypomethylation [4]. Such altered promoter DNA methylation is believed to correlate with deregulation of DNA methyltransferases and, recently, the involvement of possible demethylases is beginning to be investigated, although the molecular mechanisms implicated are still poorly understood. We investigated the involvement of COX-2 in the regulation of DNA methylation and cancer stem cell biology (fig. 1).

\section{COX-2 Impairs the Regulation of DNA Methylation}

In a previous report, Xia et al. [5] showed that prostaglandin E2 enhanced Dnmt1 and Dnmt3b protein expression, upregulated $\mathrm{CpG}$ island methylation and promoted intestinal tumor growth in ApcMin/+ mice. PGE2 enhanced Dnmt1 and Dnmt3b protein expression in colonic tumor epithelial cells and increased the number and

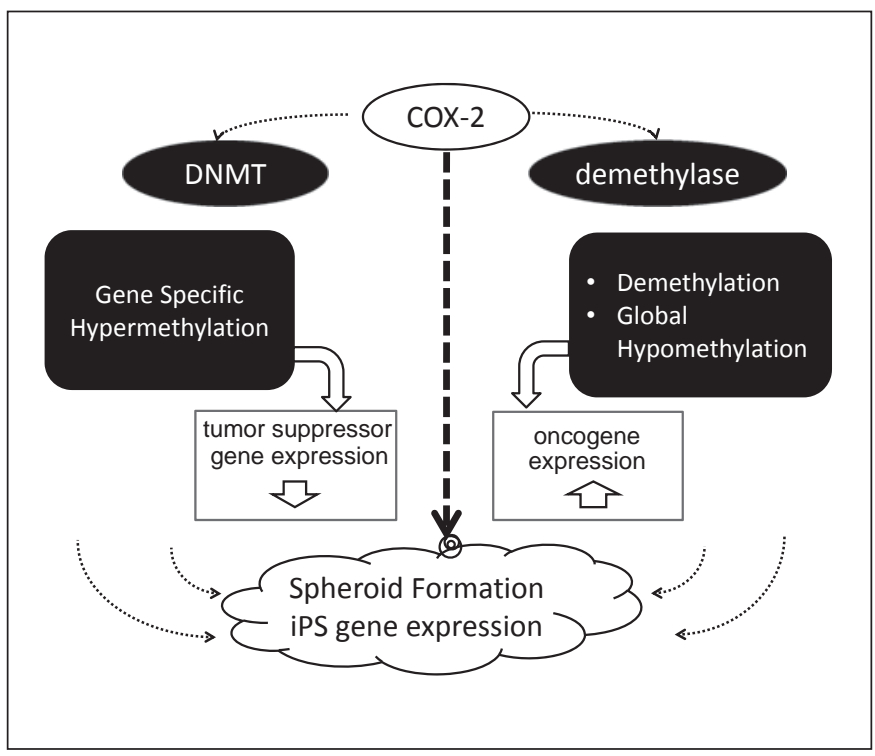

Fig. 1. Possible involvement of COX-2 in cancer stemness via epigenetic regulation. COX-2 expression is believed to affect epigenetic regulation, leading to the enhancement of cancer stemness in CRC.

size of intestinal polyps. PGE2 also decreased the expression of Cnr1 (cannabinoid receptor 1) and Mgmt (O-6methylguanine-DNA methyltransferase) at both the protein and mRNA levels in colonic tumor epithelial cells. Inhibition of $\mathrm{CpG}$ island methylation by 5 -Aza-dC attenuated PGE2-induced tumor growth in male ApcMin/+ mice. Combination treatment with celecoxib and 5-Aza$\mathrm{dC}$ more efficiently inhibited tumor growth than did treatment with either agent alone. In addition, PGE2 increased DNMT1 and DNMT3B protein expression in LS174T, a cultured cell line derived from human CRC, and blockade of PTGER4 attenuated the upregulation of DNMT1 and DNMT3B by PGE2. Moreover, knockdown of DNMT1 or DNMT3B by shRNAs attenuated the PGE2-induced downregulation of CNR1 and MGMT in LS174T cells. These results indicate that PGE2 promotes the growth of intestinal tumor via DNA methylation.

An association has been reported between COX-2 expression, hypermethylation of the RAR-beta 2 promoter region, and poor prognosis [6]. Another report has shown that celecoxib, a COX-2 inhibitor, reverses aberrant global and estrogen receptor-alpha gene methylation [7]. In our experiment investigating the effect of COX-2 on DNA methylation, COX-2 enhanced hypermethylation of tumor suppressor genes via enhanced DNMT expression. We revealed the novel finding that COX-2 reduced DNA 
methyltransferase activity and enhanced global hypomethylation and promoter hypomethylation of oncogenes. COX-2 enhanced demethylase expression, which led to accelerated aberrant hypomethylation.

These results indicate a previously unrecognized role of COX-2 in carcinogenesis via DNA methylation, although the precise mechanisms by which COX-2 can perturb epigenetic regulation remain unclear.

\section{COX-2 Plays a Pivotal Role in Cancer Stem Cell Biology}

Cancer stem cells are cited as the likely culprit of chemoresistance, metastasis and recurrence. Therefore, there is an urgent need to develop novel therapies targeting key molecules playing an important role in maintaining the properties of cancer stem cells.

Previously, it was reported that CD133, a stem cell marker, is related to COX-2 expression because CD133expressing cells showed more COX-2 expression compared with CD133-negative cells. In CD133-expressing cells, celecoxib, a COX-2-specific inhibitor, decreased radioresistance via increased phosphorylation of cdc2 [8]. Recently, it was revealed that NF-kappaB activation was involved in CD133-related COX-2 upregulation [9]. It was also reported that PGE2-mediated inflammatory signaling together with Wnt signaling triggered the expression of CD44, another stem cell marker, leading to induction of slow-cycling stem-like cells [10]. COX-2 expression was reported to be elevated in sphere-forming cells, one of the characteristic features of stem cells, and celecoxib suppressed sphere formation of CD133-expressing cells [11]. COX-2 also enhanced the expression of oct- 4 , one of the iPS-related genes and decreased Ki-67 expression, meaning suppressed cell proliferation, another characteristic feature of cancer stem cells [12].

We also investigated the effect of COX-2 on cancer stemness in cultured cell lines derived from human CRC tissues. After preparation of COX-2 expression in COX-2 non-expressing cells by genetic engineering procedure or depletion of COX-2 expression in COX-2-expressing CRC cells by siRNA, we weighed the spheroid-forming ability of COX-2-expressing cells against that of COX-2 non-expressing cells. We found that COX-2 expression was involved in the expression of iPS-related genes and COX-2-expressing cells showed more enhanced characteristics particular to stem cells, such as spheroid-forming ability, chemoresistance, cell cycle-arrest and reduced radical production, than COX-2 non-expressing cells.
COX-2-specific inhibitors suppressed the above characteristic features notably observed in COX-2-expressing cells. These results indicated that COX-2 has important roles in cancer stem cell biology.

\section{Aberrant DNA Hypermethylation and Hypomethylation Induced by COX-2 Impacts on Cancer Stem Cell Biology}

Previously, it was reported that DNA and histone methylation play a pivotal role in stem cell biology. Polycomb genes are related to DNA methylation via histone methylation and contribute to the state of cancer stem cells. Inhibition of the polycomb gene repressive complex (PRC) 1 and 2 reduces reprogramming efficiency, meaning $\mathrm{PRC} 1$ and 2 are involved in stem cell function [13].

Basically, it is contemplated that in stem cells the genes related to differentiation are silenced by DNA methylation and cancer-germline genes are silenced by DNMT3A and DNMT3B [14]. As another mechanism, it has been reported that the stem cell protein SALL4 represses gene expression through interaction with DNMTs and HDAC, and mediates stem cell self-renewal [15]. It has also been reported that DNMT1 is related to the self-renewal of stem cells, because haploinsufficiency of DNMT1 impaired its self-renewal [16], while DNMT3a silenced selfrenewal genes in stem cells [17]. In stem cells, DNA methylation inhibited the expression of differentiated cell-specific microRNA miR-122, resulting in the facilitation of self-renewal and proliferation via $\mathrm{Pkm} 2$ gene expression [18]. miR-34c was also downregulated by DNA methylation, leading to the promotion of self-renewal in tumor initiation via Notch4 expression [19]. It has also been shown that active DNA demethylation occurs during terminal specification of stem cells, leading to expression of differentiation-related genes, and that GADD45A is involved in such an essential role in gene-specific active DNA demethylation [20]. The TET protein family is reported to play an important role in terminal differentiation by converting the 5-position of cytosines to 5-hydroxymethylcytosine [21].

Hypomethylation also plays an important role in cancer stem cell function. Hypomethylation at specific CpGs in embryonic stem cells increases with cancer invasion [22]. In pluripotent stem cell-based diseases, stem cellspecific epigenetic and transcriptional aberrations are identified in genes subject to $\mathrm{X}$ chromosome inactivation and genomic imprinting [23]. The oncogene FOXM1 is 
involved in stem cell maintenance. In FOXM1-expressing cells, both aberrant promoter methylation and global hypomethylation have been observed [24].

In an experiment in which we investigated the relation between DNA methylation status and stemness, expression of both DNA methyltransferases and demethylase were increased and both region-specific hypermethylation and global hypomethylation were enhanced in spheroid-forming cultured colorectal cells, compared with non-spheroid-forming cells. Overall, it appears that DNA methylation plays an important role in maintaining stem state by suppressing the differentiation-related genes, and global hypomethylation is also involved in stem cell function.

\section{Summary}

It has already become common knowledge that DNA methylation is a key pathway in carcinogenesis and plenty of agents affecting DNA methylation have been developed. However, both hypermethylation in specific regions and global hypomethylation are involved in carcinogenesis, and even regarding region-specific hyper- methylation, promotion of some genes, for example tumor suppressor genes, are aberrantly hypermethylated whilst promotion of other genes, for example oncogenic genes, are aberrantly hypomethylated in cancer cells. Therefore, to date, demethylating agents have been used, but the development of novel reagents precisely regulating aberrant hypo- and hypermethylation is much anticipated. On the other hand, there is an urgent need to develop novel therapies targeting key molecules playing an important role in maintaining the properties of cancer stem cells. It has been reported that COX-2 is involved in chemoresistance, differential inhibition and metastasis. We found that COX-2 is involved in regulation of DNA methylation and cancer stem cell biology. Moreover, COX-2 inhibitors are anti-cancer reagents which target aberrant epigenetics and cancer stemness. This is currently facilitating the development of novel cancer therapies.

\section{Disclosure Statement}

The author declares no conflict of interest.

\section{References}

1 Lamas MJ, Duran G, Gallardo E: Anti-EGFR therapy in first-line colorectal cancer. Expert Rev Anticancer Ther 2011;11:1499-1503.

2 Kawai N, Tsujii M, Tsuji S: Cyclooxygenases and colon cancer. Prostaglandins Other Lipid Mediat 2002;68-69:187-196.

3 Buczacki S, Davies RJ, Winton DJ: Stem cells, quiescence and rectal carcinoma: an unexplored relationship and potential therapeutic target. Br J Cancer 2011;105:1253-1259.

4 Matsubara N: Epigenetic regulation and colorectal cancer. Dis Colon Rectum 2012; 55:96-104

5 Xia D, Wang D, Kim SH, Katoh H, DuBois RN: Prostaglandin E2 promotes intestinal tumor growth via DNA methylation. Nat Med 2012;18:224-226.

-6 Miladi-Abdennadher I, Abdelmaksoud-Damak R, Ayadi L, Khabir A, Frikha F, Kallel L, Amouri A, Frikha M, Sellami-Boudawara T, Gargouri A, Mokdad-Gargouri R: Hypermethylation of RAR $\beta 2$ correlates with high COX-2 expression and poor prognosis in patients with colorectal carcinoma. Tumour Biol 2010;31:503-511.
7 Shen R, Tao L, Xu Y, Chang S, Van Brocklyn J, Gao JX: Reversibility of aberrant global DNA and estrogen receptor-alpha gene methylation distinguishes colorectal precancer from cancer. Int J Clin Exp Pathol 2009;2:21-33.

8 Ma HI, Chiou SH, Hueng DY, Tai LK, Huang PI, Kao CL, Chen YW, Sytwu HK: Celecoxib and radioresistant glioblastoma-derived CD133+ cells: improvement in radiotherapeutic effects - laboratory investigation. J Neurosurg 2011;114:651-662.

$\checkmark 9$ Annabi B, Laflamme C, Sina A, Lachambre MP, Beliveau R: A MT1-MMP/NF-kappaB signaling axis as a checkpoint controller of COX-2 expression in CD133+ U87 glioblastoma cells. J Neuroinflammation 2009;6:8.

10 Ishimoto T, Oshima H, Oshima M, Kai K, Torii R, Masuko T, Baba H, Saya H, Nagano O: CD44+ slow-cycling tumor cell expansion is triggered by cooperative actions of Wnt and prostaglandin E2 in gastric tumorigenesis. Cancer Sci 2010;101:673-678.

11 Chen KH, Hsu CC, Song WS, Huang CS, Tsai CC, Kuo CD, Hsu HS, Tsai TH, Tsai CY, Woung LC, Chiou SH, Lu KH, Chen YW: Celecoxib enhances radiosensitivity in medulloblastoma-derived CD133-positive cells. Childs Nerv Syst 2010;26:1605-1612.
12 Singh B, Cook KR, Vincent L, Hall CS, Martin C, Lucci A: Role of COX-2 in tumorospheres derived from a breast cancer cell line. J Surg Res 2011;168:e39-e49.

13 Onder TT, Kara N, Cherry A, Sinha AU, Zhu N, Bernt KM, Cahan P, Marcarci BO, Unternaehrer J, Gupta PB, Lander ES, Armstrong SA, Daley GQ: Chromatin-modifying enzymes as modulators of reprogramming. Nature 2012;483:598-602.

14 Loriot A, Parvizi GK, Reister S, De Smet C: Silencing of cancer-germline genes in human preimplantation embryos: Evidence for active de novo DNA methylation in stem cells. Biochem Biophys Res Commun 2012; 417:187-191.

15 Yang J, Corsello TR, Ma Y: Stem cell gene SALL4 suppresses transcription through recruitment of DNA methyltransferases. J Biol Chem 2012;287:1996-2005.

16 Trowbridge JJ, Sinha AU, Zhu N, Li M, Armstrong SA, Orkin SH: Haploinsufficiency of Dnmt1 impairs leukemia stem cell function through derepression of bivalent chromatin domains. Genes Dev 2012;26:344-349. 
17 Trowbridge JJ, Orkin SH: Dnmt3a silences hematopoietic stem cell self-renewal. Nat Genet 2011;44:13-14.

18 Jung CJ, Iyengar S, Blahnik KR, Ajuha TP, Jiang JX, Farnham PJ, Zern M: Epigenetic modulation of miR-122 facilitates human embryonic stem cell self-renewal and hepatocellular carcinoma proliferation. PLoS One 2011;6:e27740.

19 Yu F, Jiao Y, Zhu Y, Wang Y, Zhu J, Cui X, Liu Y, He Y, Park EY, Zhang H, Lv X, Ma K, Su F, Park JH, Song E: MicroRNA 34c gene downregulation via DNA methylation promotes self-renewal and epithelial-mesenchymal transition in breast tumor-initiating cells. J Biol Chem 2012;287:465-473.
20 Zhang RP, Shao JZ, Xiang LX: GADD45A protein plays an essential role in active DNA demethylation during terminal osteogenic differentiation of adipose-derived mesenchymal stem cells. J Biol Chem 2011;286: 41083-41094.

21 Haffner MC, Chaux A, Meeker AK, Esopi DM, Gerber J, Pellakuru LG, Toubaji A, Argani $\mathrm{P}$, Iacobuzio-Donahue $\mathrm{C}$, Nelson WG, Netto GJ, De Marzo AM, Yegnasubramanian S: Global 5-hydroxymethylcytosine content is significantly reduced in tissue stem/ progenitor cell compartments and in human cancers. Oncotarget 2011;2:627-637.

22 Zhuang J, Jones A, Lee SH, Ng E, Fiegl H, Zikan M, Cibula D, Sargent A, Salvesen HB, Jacobs IJ, Kitchener HC, Teschendorff AE, Widschwendter M: The dynamics and prognostic potential of DNA methylation changes at stem cell gene loci in women's cancer. PLoS Genet 2012;8:e1002517.
23 Nazor KL, Altun G, Lynch C, Tran H, Harness JV, Slavin I, Garitaonandia I, Muller FJ, Wang YC, Boscolo FS, Fakunle E, Dumevska B, Lee S, Park HS, Olee T, D'Lima DD, Semechkin R, Parast MM, Galat V, Laslett AL, Schmidt U, Keirstead HS, Loring JF, Laurent LC: Recurrent variations in DNA methylation in human pluripotent stem cells and their differentiated derivatives. Cell Stem Cell 2012;10:620-634.

24 Teh MT, Gemenetzidis E, Patel D, Tariq R, Nadir A, Bahta AW, Waseem A, Hutchison IL: Foxm1 induces a global methylation signature that mimics the cancer epigenome in head and neck squamous cell carcinoma. PLoS One 2012; 7:e34329. 